\title{
Towards DMRG-tailored coupled cluster method in the 4c-relativistic domain
}

\author{
Jan Brandejs, ${ }^{1,2, a)}$ Jakub Višňák, ${ }^{1,2,3, b)}$ Libor Veis, ${ }^{1,(\mathrm{c})}$ Maté Mihály, ${ }^{4,5, \mathrm{~d})}$ Örs Legeza, ${ }^{4, e)}$ and Jiří Pittner ${ }^{1, f)}$ \\ 1) J. Heyrovsky Institute of Physical Chemistry, Academy of Sciences of the Czech Republic, v.v.i., Dolejškova 3, 18223 Prague 8, \\ Czech Republic \\ 2) Faculty of Mathematics and Physics, Charles University, Prague, Czech Republic \\ ${ }^{3)}$ Czech Academic City in Erbil, Yassin Najar street, Kurani Ankawa, Erbil, Kurdistan Region of Iraq \\ ${ }^{4)}$ Strongly Correlated Systems "Lendület" Research Group, Institute for Solid State Physics and Optics, \\ MTA Wigner Research Centre for Physics, H-1121 Budapest, Konkoly-Thege Miklós út 29-33, \\ Hungary \\ ${ }^{5)}$ Department of Physics of Complex Systems, Eötvös Loránd University, Pf. 32, H-1518 Budapest, \\ Hungary
}

(Dated: 16 April 2020)

There are three essential problems in computational relativistic chemistry: electrons moving at relativistic speeds, close lying states and dynamical correlation. Currently available quantum-chemical methods are capable of solving systems with one or two of these issues. However, there is a significant class of molecules, in which all the three effects are present. These are the heavier transition metal compounds, lanthanides and actinides with open $\mathrm{d}$ or $\mathrm{f}$ shells. For such systems, sufficiently accurate numerical methods are not available, which hinders the application of theoretical chemistry in this field. In this paper, we combine two numerical methods in order to address this challenging class of molecules. These are the relativistic versions of coupled cluster methods and density matrix renormalization group (DMRG) method. To the best of our knowledge, this is the first relativistic implementation of the coupled cluster method externally corrected by DMRG. The method brings a significant reduction of computational costs, as we demonstrate on the system of $\mathrm{TlH}, \mathrm{AsH}$ and $\mathrm{SbH}$.

\section{INTRODUCTION}

At the turn of the millennium, the density matrix renormalization group method (DMRG) $)^{\sqrt{1}}$ was introduced to quantumchemical community 2 and since then, it has seen a large surge in the use for multireference systems. The biggest advantage of DMRG method is its capability to treat large active spaces, current implementations can go to about 50 active space spinors $5 \sqrt{6}$. However, a major drawback of DMRG is its inability to capture dynamical correlation, since it cannot include all virtual spinors. This correlation has a strong influence in the target systems of this project, which thus aims to address this problem. The DMRG method is already well established and computational chemists started to use it, however, the methods for treating the dynamical correlations on top of DMRG are still in pioneering stage. Past efforts were either based on second order perturbation theory ${ }^{7}$, internally contracted MRCI (multireference configuration interaction $) \sqrt{8}$, random phase approximation $\frac{9}{\text {, canonical }}$ transformation method 10 , or the perturbation theory with matrix product states 11 .

Our group has followed a different pathway to deal with the dynamical correlation, the coupled cluster method exter-

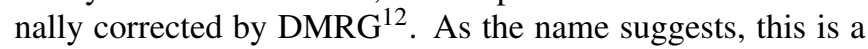

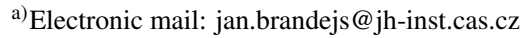

b)Electronic mail: jakub.visnak@jh-inst.cas.cz



d)Electronic mail: mate.mihaly@wigner.mta.hu

e)Electronic mail: legeza.ors@wigner.mta.hu

${ }^{\mathrm{f}}$ Electronic mail: jiri.pittner@jh-inst.cas.cz
}

combination of DMRG and the coupled cluster (CC) method. The CC method is known for its ability to describe dynamical correlation. In the externally corrected approach, first a DMRG calculation is performed on the strongly correlated active space, keeping the rest of the system fixed. This accounts for the static correlation. Second step is CC analysis of matrix product state (MPS) wave function, obtained from DMRG. Then a CC calculation is performed on the rest of the system, keeping in turn the active space amplitudes fixed, which captures the dynamical correlation. Already the simplest version thereof, the tailored CCSD (CC with single and double excitations) approach ${ }^{13 \mid 14}$, yields very promising results 12 . Remarkably, all previous approaches based on the use of DMRG output in another method have so far been non-relativistic, leaving the relativistic domain unexplored. This is the focus of this paper.

First we demonstrate the capabilities of our relativistic 4cTCCSD implementation on the example of the thallium hydride $(\mathrm{TlH})$ molecule, which has become a standard benchmark molecule for relativistic methods and most importantly large-scale DMRG and up to CCSDTQ results are available ${ }^{15}$. It should be noted that DMRG is best suited for staticcorrelation problems while $\mathrm{TlH}$ is dominated mostly by dynamic correlation, for which $\mathrm{CC}$ approaches are excellent. In order to study the behaviour of TCCSD method for more multireference systems, we performed tests on $\mathrm{AsH}$ and $\mathrm{SbH}$ molecules. The multireference character in $\mathrm{AsH}$ and $\mathrm{SbH}$ ground state arises from the fact that two determinants are needed to describe $\mathrm{M}_{s}=0$ triplet component in the spin-free case, and therefore is somewhat "artificial". Figure 1 depicts the $\pi_{1 / 2}^{2}$ and $\pi_{3 / 2}^{2}$ determinants arising from $M_{s}=0$ triplet component's determinants due to spin-orbit splitting for $\mathrm{AsH}$ 
and $\mathrm{SbH}$. The heavier the atom is, the greater the splitting, then $\pi_{1 / 2}^{2}$ is more dominant and $\mathrm{X}^{+}$ground state becomes less multireference. Hence we expect $\mathrm{AsH}$ to be of stronger multireference nature than $\mathrm{SbH}$.

$$
\begin{aligned}
& |\uparrow \uparrow\rangle \stackrel{M=1}{\longrightarrow} \mathrm{C}_{1} "|+\rangle \mathrm{AA}
\end{aligned}
$$

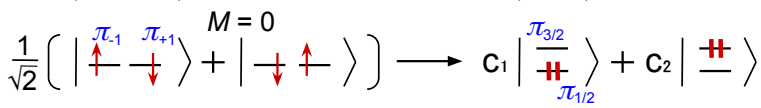

$$
\begin{aligned}
& |\downarrow \downarrow\rangle) \stackrel{M=-1}{\longrightarrow} \mathrm{C}_{1}^{\prime \prime}|+\rangle \mathrm{BB}
\end{aligned}
$$

FIG. 1: Spin-orbit splitting in $\mathrm{AsH}$ and $\mathrm{SbH}$.

\section{THEORY}

Present-day relativistic calculations are often carried out within the no-pair approximation, where the Dirac-Coulomb Hamiltonian is embedded by projectors eliminating the troublesome negative-energy solutions, which yields a second quantized Hamiltonian formally analogous to the nonrelativistic case

$$
H=\sum_{P Q} h_{P}^{Q} a_{P}^{\dagger} a_{Q}+\frac{1}{4} \sum_{P Q R S}\langle P Q \| R S\rangle a_{P}^{\dagger} a_{Q}^{\dagger} a_{S} a_{R},
$$

where the indices $P, Q, R, S$ run over the positive-energy 4component spinors spanning the one-electron basis. The barred spinors $\left(\phi_{\bar{p}}\right)$ and unbarred spinors $\left(\phi_{p}\right)$ form Kramers pairs related to each other by action of the time-reversal operator $K$

$$
\begin{aligned}
& K \phi_{p}=\phi_{\bar{p}}, \\
& K \phi_{\bar{p}}=-\phi_{p} .
\end{aligned}
$$

The Kramers symmetry replaces the spin symmetry in the non-relativistic theory; in particular $M_{S}$ is not a good quantum number and $M_{K}$ projection is defined instead, which is $1 / 2$ for unbarred spinors (A) and $-1 / 2$ for spinors with barred indices (B). The capital indices in (1) run over both spinors of a Kramers pair. In contrast to the non-relativistic case, the Hamiltonian (1) is in general not block-diagonal in $M_{K}$. Since each creation or annihilation operator in (1) changes $M_{K}$ by $\pm 1 / 2$, the Hamiltonian couples states with $\left|\Delta M_{K}\right| \leq 2$. Moreover, the index permutation symmetry of the 2e-integrals in (1) is lower than in the non-relativistic case.

The Dirac program 16 employs a quaternion symmetry approach which combines the Kramers and binary double group symmetry $\left(D_{2 h}^{*} \text { and subgroups }\right)^{17}$. The double groups can be sorted into three classes based on the application of the Frobenius-Schur indicator to their irreducible representations: "real groups" $\left(D_{2 h}^{*}, D_{2}^{*}\right.$, and $\left.C_{2 v}^{*}\right)$; "complex groups" $\left(C_{2 h}^{*}\right.$, $C_{2}^{*}$, and $\left.C_{s}^{*}\right)$; and "quaternion groups" $\left(C_{i}^{*}\right.$ and $\left.C_{1}^{*}\right)$. Generalization of non-relativistic methods is simplest in the "real groups" case, where the integrals are real-valued and the ones with odd number of barred (B) indices vanish. In practice, it means that additional "spin cases" of integrals $(A B \mid A B)$ and (AB|BA) (in Mulliken notation) have to be included. For the complex groups, the integrals are complex-valued, but still only integrals with even number of barred indices are nonzero. Finally, in the remaining case of "quaternion groups" all the integrals have to be included and are complex-valued 18 .

The idea of externally corrected coupled cluster methods is to take information on static correlation from some nonCC external source, and to include it into the subsequent CC treatment ${ }^{20}$. The conceptually simplest approach is the tailored CC method (TCC) proposed by Bartlett et al.13/21-23, which uses the split-amplitude ansatz for the wave function introduced by Piecuch et al.24[25

$$
|\Psi\rangle=e^{T_{\mathrm{ext}}} e^{T_{\mathrm{cas}}}|\Phi\rangle
$$

where $T_{\text {cas }}$ containing amplitudes with all active indices is "frozen" at values obtained from CASCI or in our case from DMRG. The external cluster operator $T_{\text {ext }}$ is composed of amplitudes with at least one index outside the CAS space. Another way to justify this ansatz is the formulation of $\mathrm{CC}$ equations based on excitation subalgebras recently introduced by Kowalski26. The simplest version of the method truncates both $T_{\text {cas }}$ and $T_{\text {ext }}$ to single and double excitations. Since there is a single-determinantal Fermi vacuum, the excitation operators $T_{\text {ext }}$ and $T_{\text {cas }}$ commute, which keeps the method very simple. TCC can thus use the standard CCSD solver, modified to keep the amplitudes from $T_{\text {cas }}$ fixed. Thanks to the two-body Hamiltonian, tailored CCSD energy with the $T_{\text {ext }}=0$ and $T_{\text {cas }}$ from CASCI (complete active space configuration interaction) reproduces the CASCI energy. In the limit of CAS space including all MOs, TCC thus recovers the FCI energy. In general, a quadratic error bound valid for TNS-TCC methods is derived 27 .

In 1228 we have described how to obtain $T_{\text {cas }}$ from the DMRG wave function using concepts of quantum information theory ${ }^{29}$ in the non-relativistic case, yielding the DMRGTCCSD method. The DMRG method ${ }^{30}$ is a procedure which variationally optimizes the wave function in the form of the matrix product state (MPS) ansat $\mathrm{z}^{5}$. The quantum chemical version of DMRG (QC-DMRG) $\sqrt[31+36]{\text { eventually converges to }}$ the FCI solution in a given orbital space, i.e. to CASCI. The practical version of DMRG is the two-site algorithm, which provides the wave function in the two-site MPS form ${ }^{[5}$

$$
\left|\Psi_{\mathrm{MPS}}\right\rangle=\sum_{\{\alpha\}} \mathbf{A}^{\alpha_{1}} \mathbf{A}^{\alpha_{2}} \cdots \mathbf{W}^{\alpha_{i} \alpha_{i+1}} \cdots \mathbf{A}^{\alpha_{n}}\left|\alpha_{1} \alpha_{2} \cdots \alpha_{n}\right\rangle
$$

where $\alpha_{i} \in\{|0\rangle,|\downarrow\rangle,|\uparrow\rangle,|\downarrow \uparrow\rangle\}$ and for a given pair of adjacent indices $[i,(i+1)], \mathbf{W}$ is a four index tensor, which corresponds to the eigenfunction of the electronic Hamiltonian expanded in the tensor product space of four tensor spaces defined on an ordered orbital chain, so called left block $\left(M_{l}\right.$ dimensional tensor space), left site (four dimensional tensor space of $i^{\text {th }}$ orbital), right site (four dimensional tensor space of $(i+1)^{\text {th }}$ orbital), and right block $\left(M_{r}\right.$ dimensional tensor space).

When employing the two-site MPS wave function (Eq. 4) for the purposes of the TCCSD method, the CI expansion co- 
efficients $c_{i}^{a}$ and $c_{i j}^{a b}$ for $a, b, i, j \in \mathrm{CAS}$ can be efficiently calculated by contractions of MPS matrices 3738 . We would like to note that using the two-site DMRG approach in practice means using the wave-function calculated at different sites and it can only be employed together with the dynamical block state selection (DBSS) procedure ${ }^{4}$ assuring the same accuracy along the sweep. Alternatively, one can use the onesite approach in the last sweep 39 .

Once the CI coefficients $c_{i}^{a}$ and $c_{i j}^{a b}$ have been obtained, the standard CC analysis is performed to convert them to the CC amplitudes

$$
\begin{aligned}
T_{\mathrm{CAS}}^{(1)} & =C^{(1)}, \\
T_{\mathrm{CAS}}^{(2)} & =C^{(2)}-\frac{1}{2}\left[C^{(1)}\right]^{2} .
\end{aligned}
$$

The generalization of the DMRG-TCCSD method to the relativistic $4 \mathrm{c}$ case has to consider several points. First of all, the additional integral classes with nonzero $\Delta M_{K}$ have to be implemented in the DMRG Hamiltoniar $15 / 40$. Secondly, there will be more $\mathrm{CI}$ coefficients and subsequently $\mathrm{CC}$ amplitudes to be obtained from the MPS wave function, corresponding to excitations with nonzero $\Delta M_{K}$. Finally, except for the "real groups", the DMRG procedure has to work with complex matrices and the resulting cluster amplitudes will also be complex-valued. In the present work, we have selected numerical examples with "real groups" symmetry, while the complex generalization of the DMRG code is in progress.

\section{COMPUTATIONAL DETAILS}

In the present work we have used the two-site DMRG variant together with DBSS through the course of the whole DMRG procedure and obtained the CC amplitudes from the resulting two-site form of MPS wave function. For all systems, DMRG calculations were performed exclusively with the QC-DMRG-Budapest program 41. Dirac program package ${ }^{16}$ was used for the remaining relativistic calculations, whereas Orca program was used for remaining non-relativistic calculations. Orbitals and MO integrals were generated with the Dirac program package 16 We used the Dirac-Coulomb Hamiltonian and triple-zeta basis sets for the heavier of the two atoms (cv3z for Tl, As and Sb and cc-pVTZ for F) as well as for hydrogen (cc-pVTZ), which include core-correlating functions for the heavier atom. Initialization of DMRG, i.e., optimal ordering of spinors, was set up as discussed in Ref. 35. The numerical accuracy was controlled by $\mathrm{DBSS}^{4}$ keeping up to thousands of block states for the a priori set quantum information loss threshold $\chi=10^{-6}$.

\section{A. Comparison with non-relativistic TCC}

In order to compare with non-relativistic version of TCCSD method, the system of hydrogen fluoride was chosen, as it is a biatomic with light nuclei. CC-pVTZ basis was used at the internuclear distance of $0.8996 \AA$.
Consistent methods should exhibit a constant shift of relativistic and non-relativistic energy $\Delta E=E_{\text {rel }}-E_{\text {nonrel, }}$, given by a different Hamiltonian. Table I shows that TCCSD is consistent with RHF, CCSD and DMRG methods in terms of $\Delta E$ up to a millihartree.

\begin{tabular}{|r|r|r|r|}
\hline method & $E_{\text {rel }}\left[\mathrm{E}_{h}\right]$ & $E_{\text {nonrel }}\left[\mathrm{E}_{h}\right]$ & $\Delta E\left[\mathrm{E}_{h}\right]$ \\
\hline RHF & -100.14972 & -100.05846 & -0.09126 \\
DMRG(6,6) & -100.15868 & -100.06737 & -0.09130 \\
CCSD & -100.42322 & -100.33172 & -0.09150 \\
TCCSD(6,6) & -100.42418 & -100.33246 & -0.09172 \\
\hline
\end{tabular}

TABLE I: Comparison of energies of HF molecule obtained from relativistic and non-relativistic methods. The rightmost column shows the difference between the $4 \mathrm{c}$-relativistic energy $E_{\text {rel }}$ and non-relativistic energy $E_{\text {nonrel }}$.

\section{B. TIH}

We have used the computational protocol of Ref. 15 for direct comparison with their energies. $\mathrm{C}_{2 \mathrm{v}}^{\star}$ double group symmetry with real irreps was assumed. The 4c-RHF energy was -20275.41661 $\mathrm{E}_{h}$. We used MP2 natural spinors (NS) from the Dirac program 16 as the spinor basis for electroncorrelation calculations, correlating the $\mathrm{Tl} 5 \mathrm{~s}, 5 \mathrm{p}, 4 \mathrm{f} 5 \mathrm{~d}, 6 \mathrm{~s}$, $6 \mathrm{p}$ and $\mathrm{H}$ 1s electrons while keeping the remaining core electrons of $\mathrm{Tl}$ frozen. Using uncontracted basis, a virtual spinor threshold was set at $135 \mathrm{E}_{h}$. The resulting space $(14,47)$ was chosen by ordering MP2 NS by their occupations and taking those with values between 1.98 and 0.001 . In this space, the $4 \mathrm{c}-\mathrm{TCCSD}$ was performed, with DMRG calculations in the procedure limited to subspaces of $(14,10),(14,14),(14,17)$, $(14,25)$ and $(14,29)$, with spinors sorted by MP2 occupations. Figure 2 shows a scheme of embedded active spaces used in the procedure.



FIG. 2: Schematic depiction of active spaces used in the 4-TCCSD procedure for $\mathrm{TlH}$.

\section{AsH and $\mathrm{SbH}$}

Since $\mathrm{SbH}$ is a heavier homologue of AsH (which is itself homologue of nitrene, $\mathrm{NH}$ ), the procedure was very similar for both of them. In contrast with TlH, instead of MP2 NS, we used average-of-configuration SCF spinors. The 4c-SCF energy was $-2260.04261 \mathrm{E}_{h}$ for AsH and -6481.10775 $\mathrm{E}_{h}$ for $\mathrm{SbH}$. The DMRG calculation in the 4c-TCCSD procedure was limited to subspaces of $(16,14)$ and $(16,23)$. 
Dominant contributions to active spaces for AsH active space $(16,14)$ are As: $3 \mathrm{~d}, 4 \mathrm{~s}, 4 \mathrm{p}, 5 \mathrm{~s}, 5 \mathrm{p}, \mathrm{H}: 1 \mathrm{~s}$ and for active space $(16,23)$ we add As: $4 d, H: 2 s, 2 p$ to the former. Energies of MOs are: from $-2.1 \mathrm{E}_{h}$ to $+0.25 \mathrm{E}_{h}$ and $+1.01 \mathrm{E}_{h}$ for $(16,14)$ and $(16,23)$ respectively (for internuclear distance $1.52 \AA$ ). Dominant contributions to active spaces for $\mathrm{SbH}$ are analogous, except for principal quantum numbers, which are higher by 1 .

\section{RESULTS AND DISCUSSION}

\begin{tabular}{|c|c|c|}
\hline method & $E_{\mathrm{el}}\left[\mathrm{E}_{h}\right]$ & $\Delta E_{\mathrm{el}}\left[\mathrm{mE}_{h}\right]$ \\
\hline 4c-MP2(14,47) & -20275.85372 & -13.49 \\
\hline $4 c-\operatorname{CCSD}(14,47)$ & -20275.82966 & 10.58 \\
\hline $4 \mathrm{c}-\operatorname{CCSD}(\mathrm{T})(14,47)$ & -20275.84056 & -0.32 \\
\hline $4 c-D M R G(14,47)^{a} 15$ & -20275.83767 & 2.57 \\
\hline $4 c-\operatorname{TCCSD}(14,10)$ & -20275.83042 & 9.83 \\
\hline 4c-TCCSD $(14,11)$ & -20275.83170 & 8.54 \\
\hline $4 \mathrm{c}-\operatorname{TCCSD}(14,12)$ & -20275.83257 & 7.67 \\
\hline 4c-TCCSD $(14,13)$ & -20275.83329 & 6.95 \\
\hline 4c-TCCSD $(14,14)$ & -20275.83430 & 5.94 \\
\hline 4c-TCCSD $(14,15)$ & -20275.83224 & 8.00 \\
\hline 4c-TCCSD $(14,16)$ & -20275.82902 & 11.23 \\
\hline $4 c-\operatorname{TCCSD}(14,17)$ & -20275.82405 & 16.19 \\
\hline
\end{tabular}

TABLE II: Total electronic energy and energy differences $\Delta E_{\mathrm{el}}$ (in $\mathrm{mE}_{h}$ ) for various methods with respect to the 4c-CCSDTQ $(14,47)$ reference energy of $-20275.84024233 \mathrm{E}_{h}^{\sqrt{15}}$ for TIH at the experimental equilibrium internuclear distance $1.872 \AA$.

Once we reproduced the MP2 and CCSD energy of TlH in equilibrium geometry from Ref. 15, we applied the 4c-TCCSD method. Obtained energies and their respective deviations from the reference CCSDTQ calculation ${ }^{15}$ are listed in Table III In case of the optimal selection of active space of 14-spinors, the TCCSD method improved the CCSD energy by $4.94 \mathrm{mE}_{H}$. While TCCSD introduces only a minor computational cost increase over CCSD, it cuts the energy error in half. This shows the practical advantage of the method. The energy obtained by TCCSD is comparable even with large-scale DMRG in the full CAS $(14,47)$.

As we can see from the high accuracy of the $4 c-\operatorname{CCSD}(T)$ energy, the system does not exhibit a considerable multireference character. Therefore even a rather small CAS of 14 spinors is sufficient for a good description of the system.

As shown on the chart in Figure 3a, TCCSD significantly improves DMRG energy towards FCI, even for the smallest CAS space. In fact, further enlarging of CAS over the size of 14 spinors is counter productive. Although the TCC must reproduce FCI energy when CAS is extended to all spinors, the TCC energy does not approach this limit monotonically $\sqrt{42}$. The obvious reason is that the "frozen" $T_{\mathrm{CAS}}$ amplitudes cannot reflect the influence of the dynamical correlation in the external space back on the active CAS space, therefore extending CAS space first exacerbates the results. Unfortunately,

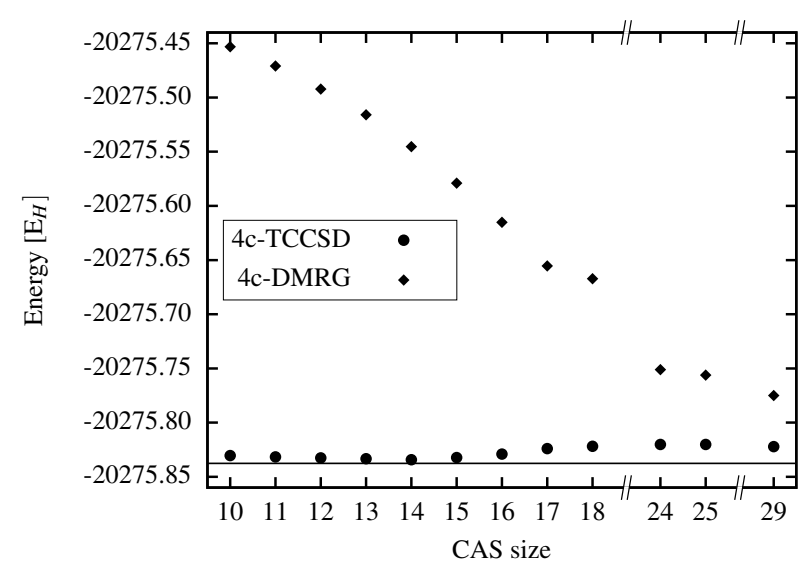

(a) Comparison of TCCSD and DMRG methods. The horizontal solid line represents the "FCI-limit" from the large 4c-DMRG( $(14,47, \sqrt{15}$ calculation.



(b) Detail of 4c-TCCSD energies.

FIG. 3: Equilibrium energy of TlH calculated using the 4c-TCCSD and 4c-DMRG methods with different sizes of DMRG active space, as given in Table

more detailed understanding of this highly nonlinear behavior ia still an open problem. In spite of a considerable effort, so far no quantities able to predict the optimal CAS-EXT split a priori have been identified ${ }^{42}$. Nevertheless, we have found that an error minimum can be obtained by sweeping through the entire orbital space with low cost DMRG calculations and this minimum does not shift or shifts only a little when more accurate calculations are performed. Therefore, in practice, the optimal CAS size related to the energy minimum is usually independent of $\mathrm{M}$ and can be determined with a low bond dimension (M) DMRG calculations ${ }^{42}$.

As demonstrated by the chart $3 \mathrm{~b}$, the optimal CAS size is 14 spinors for the equilibrium energy calculation. This CAS size is optimal not only for energies, but also for the calculation of spectroscopic properties, including the low bond dimension calculations with $M=512$ (see Table III).

Table III shows the obtained spectroscopic properties of TlH. Even for a small active space of 10 spinors, TCCSD shows an agreement with the experiment comparable with the large DMRG(14,47) calculation. For the 14-spinor space, 
spectroscopic constants obtained by TCCSD exhibit the best agreement with the experiment, thus being consistent with the lowest energy single point result of 14-spinor space in Table II. Moreover, TCCSD based on DMRG with $\mathrm{M}=512$ states or on DMRG with DBSS yield very similar results, indicating that the underlying DMRG is well converged. However, for 17-spinors, there is a bigger difference and $\mathrm{M}=512$ might not be accurate enough. This is in accordance with the previous findings of dynamical correlation effects.

\begin{tabular}{|c|c|c|c|}
\hline method & $r_{e}[\AA]$ & $\omega_{e}\left[\mathrm{~cm}^{-1}\right]$ & $\omega_{e} x_{e}\left[\mathrm{~cm}^{-1}\right]$ \\
\hline experiment $\sqrt{\mathrm{a}}$ & 1.872 & 1391 & 22.7 \\
\hline 4c-DMRG $(14,4 7 \longdiv { 1 5 }$ & 1.873 & 1411 & 26.6 \\
\hline $4 c-\operatorname{CCSD}(14,47) 15$ & 1.871 & 1405 & 19.4 \\
\hline $4 c-T C C S D(14,10)$ DBSS & 1.874 & 1404 & 24.6 \\
\hline $4 c-\operatorname{TCCSD}(14,10){ }^{\overline{0}} \mathrm{M}=512$ & 1.874 & 1403 & 23.4 \\
\hline $4 \mathrm{c}-\mathrm{TCCSD}(14,14)^{\overline{\vec{D}}} \mathrm{DBSS}$ & 1.869 & 1412 & 22.6 \\
\hline $4 \mathrm{c}-\mathrm{TCCSD}(14,14) \mathrm{DBSS}$ & 1.869 & 1411 & 20.1 \\
\hline $4 \mathrm{c}-\operatorname{TCCSD}(14,14)^{\sqrt{\vec{D}}} \mathrm{M}=512$ & 1.869 & 1411 & 22.6 \\
\hline $4 c-\operatorname{TCCSD}(14,14) \mathrm{M}=512$ & 1.869 & 1411 & 20.3 \\
\hline $4 c-\operatorname{TCCSD}(14,17)$ DBSS & 1.859 & 1426 & 20.5 \\
\hline $4 c-\operatorname{TCCSD}(14,17)$ DBSS & 1.859 & 1426 & 17.5 \\
\hline $4 c-\operatorname{TCCSD}(14,17)^{\bar{D}} \mathrm{M}=512$ & 1.859 & 1428 & 22.4 \\
\hline $4 c-\operatorname{TCCSD}(14,17) \cdot \mathrm{M}=512$ & 1.859 & 1428 & 29.8 \\
\hline
\end{tabular}

${ }^{a}$ GRECP spin-orbit MRD-CI, see Ref. 43.

$\mathrm{b}$ TWOFIT 4th order polynomial.

c VIBANAL 10th order polynomial. $\mathrm{R}_{\min }-\mathrm{R}_{\max }$ [ $\left.\mathrm{A}\right]$ : $1.64-2.20$ for $(14,14)$; 1.70-2.04 for $(14,17)$ DBSS and 1.72-2.00 for $(14,17) \mathrm{M}=512$.

TABLE III: Spectroscopic constants of ${ }^{205} \mathrm{TlH}$ obtained from 4c-TCCSD, compared with calculations and experimental work from the literature. The spectroscopic constants have been evaluated from potential energy curve fit, with two different methodologies. In case of TWOFIT methodology, the number of points have been selected according to Mean displacement in harmonic ground state criterion. In case of VIBANAL methodology, a wider symmetric interval around equilibrium geometry has been selected. In all cases, internuclear separation axis sampling was chosen to be $0.02 \AA$.

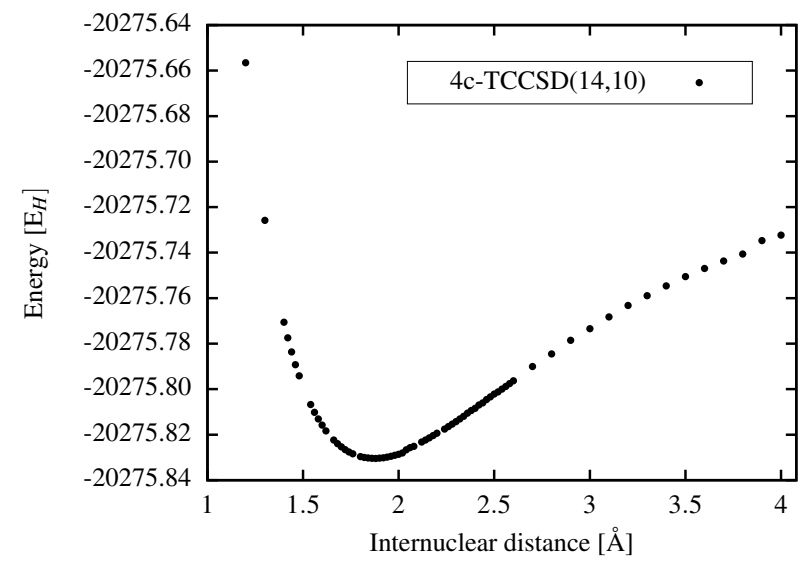

FIG. 4: Dissociation curve of $\mathrm{TlH}$.

In order to further assess the feasibility of the method for multireference systems, we studied $\mathrm{AsH}$ and $\mathrm{SbH}$ molecules. Table IV compares the determinants with highest coefficients as calculated by $4 \mathrm{c}-\mathrm{DMRG}$ with the space of $(14,14)$ for $\mathrm{TlH}$ and $(16,14)$ for $\mathrm{AsH}$ and $\mathrm{SbH}$. The coefficients confirm that the need arises for a multireference description of the ground state of $\mathrm{AsH}$ and that $\mathrm{SbH}$ is between $\mathrm{AsH}$ and $\mathrm{TlH}$ in terms of its multireference character.

The equilibrium energies of AsH in Table $\mathrm{V}$ show that $4 \mathrm{c}$ TCCSD improved the 4c-CCSD by $17 \mathrm{mE}_{h}$, with just $3 \mathrm{mE}_{h}$ difference from $4 \mathrm{c}-\mathrm{CCSD}(\mathrm{T})$. In this case a more accurate theoretical reference energy is unavailable, and therefore we turned to spectroscopic constants, as shown in Table VI, to enable a comparison with accurate IR spectra obtained by $\mathrm{CO}$ laser magnetic resonance in Ref. 44. The respective potential curve is plotted in Figure 5. The comparison of both the internuclear distance $r_{e}$ and the vibrational constant $\omega_{e}$ shows a clear advantage of 4c-TCCSD over 4c-CCSD in this case, which we attribute to the multireference nature of AsH. Despite the multireference character, the 4c-CCSD(T) with perturbative triples still prevails over 4c-TCCSD. This shows that at this range of internuclear distances the multireference character is not strong enough to cause CCSD(T) to fail. Nevertheless, in AsH it is strong enough that TCCSD provides a major improvement over CCSD at the same computational scaling.

\begin{tabular}{|c|c|c|}
\hline & determinant & coefficient \\
\hline \multirow[t]{4}{*}{$\mathrm{TlH}$} & 22222220000000 & reference \\
\hline & 22222220000000 & 0.97862 \\
\hline & 22222202000000 & 0.05928 \\
\hline & 22222200200000 & 0.05540 \\
\hline \multirow[t]{4}{*}{$\mathrm{SbH}$} & 22222222000000 & reference \\
\hline & 22222222000000 & 0.82518 \\
\hline & 22222220200000 & 0.54748 \\
\hline & 22222202020000 & 0.04872 \\
\hline \multirow[t]{4}{*}{ AsH } & 22222222000000 & reference \\
\hline & 22222222000000 & 0.75673 \\
\hline & 22222220200000 & 0.64048 \\
\hline & 22222202020000 & 0.04123 \\
\hline
\end{tabular}

TABLE IV: Three configurations with the highest coefficients (in absolute values) for $\mathrm{TlH}, \mathrm{SbH}$ and $\mathrm{AsH}$ in equilibrium internuclear distance, as generated by DMRG with the active space of 14 spinors. In each case, the $\mathrm{CC}$ amplitudes were generated with respect to the closed shell reference listed in the first row for given system. Here 2 is for a doubly occupied Kramers pair and 0 is for an empty Kramers pair.

\begin{tabular}{|l|r|}
\hline method & $E_{\mathrm{el}}\left[\mathrm{E}_{h}\right]$ \\
\hline 4c-MP2(16,81) & -2260.53227 \\
4c-CCSD $(16,81)$ & -2260.53241 \\
4c-TCCSD $(16,14)$ & -2260.54945 \\
4c-CCSD $(\mathrm{T})(16,81)$ & -2260.55133 \\
\hline
\end{tabular}

TABLE V: Total energy for various methods for AsH at the experimental equilibrium internuclear distance ${ }^{45}$ of $1.5343 \AA$. 


\begin{tabular}{|c|c|c|c|}
\hline method & $r_{e}[\AA]$ & $\omega_{e}\left[\mathrm{~cm}^{-1}\right]$ & $\omega_{e} x_{e}\left[\mathrm{~cm}^{-1}\right]$ \\
\hline experiment & 1.523 & 2156 & 39.2 \\
\hline $4 \mathrm{c}-\mathrm{SCF}$ & 1.513 & 2382 & 33.6 \\
\hline $4 c-M P 2(16,81)^{e}$ & 1.503 & 2256 & 35.0 \\
\hline 4c-DMRG(16,14) DBSS & 1.545 & 2051 & 42.2 \\
\hline $4 c-\operatorname{CCSD}(16,81)$ & 1.505 & 2281 & 40.0 \\
\hline $4 \mathrm{c}-\mathrm{TCCSD}(16,14)^{\mathrm{d}} \mathrm{DBSS}$ & 1.517 & 2154 & 43.9 \\
\hline 4c-TCCSD $(16,14)$ DBSS & 1.517 & 2172 & 39.6 \\
\hline $4 c-\operatorname{CCSD}(T)(16,81)^{\mathrm{e}}$ & 1.521 & 2146 & 40.6 \\
\hline
\end{tabular}

e TWOFIT 4th order polynomial.

d VIBANAL 10th order polynomial.

TABLE VI: Spectroscopic constants of ${ }^{75} \mathrm{AsH}$ obtained from 4c-TCCSD, compared with calculations and experimental work from the literature.

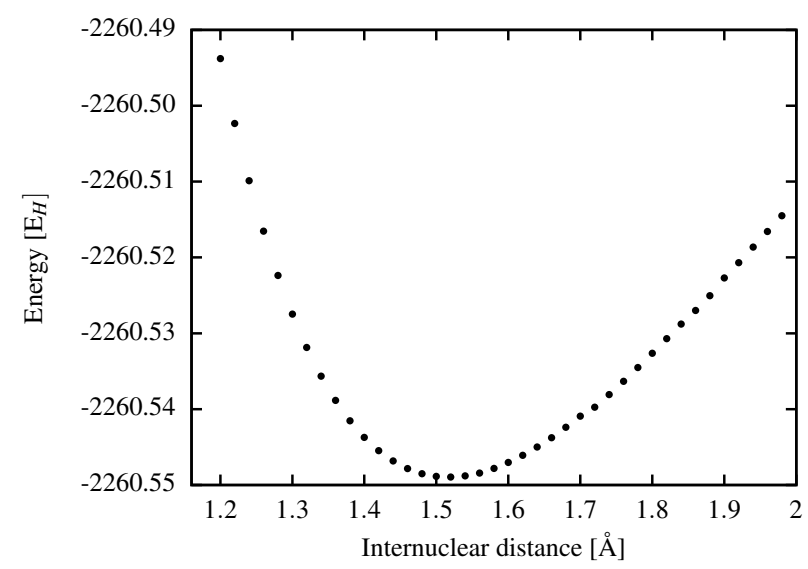

FIG. 5: Detail of potential curve of AsH near the equilibrium internuclear distance.

Calculated equilibrium energies for $\mathrm{SbH}$ are listed in Table VII Compared with the higher order methods, the 4c-MP2 method outputs lower energy, which might be due to a failure to describe the multireference character of this system. As with the previous systems, TCCSD energy is between CCSD and $\operatorname{CCSD}(\mathrm{T})$. However, in constrast with $\mathrm{TlH}$ where static correlation was not essential for a good description, he it is not clear if $\operatorname{CCSD}(\mathrm{T})$ is a good benchmark, since for multireference systems CCSD(T) tends to output too low energy. Unfortunately, we miss a more accurate calculation to compare with, hence we again turn to spectroscopic constants, which are listed in Table VIII Considering the calculated internuclear distance, MP2 and DMRG are inaccurate, as they were for AsH. Oddly enough, plain DMRG with a small active space is in both cases very accurate for $\omega_{e}$ and $\omega_{e} x_{e}$. Methods from CC theory succeeded in describing $r_{e}, \omega_{e}$ and $\omega_{e} x_{e}$, and TCCSD again outputs values between CCSD and CCSD(T). Compared with CCSD, TCCSD improved the vibrational constant $\omega_{e}$.

\begin{tabular}{|l|r|}
\hline method & $E_{\mathrm{el}}\left[\mathrm{E}_{h}\right]$ \\
\hline 4c-MP2(16,81) & -6481.69651 \\
4c-CCSD(16,81) & -6481.67108 \\
4c-TCCSD(16,14) & -6481.68380 \\
4c-CCSD(T)(16,81) & -6481.69315 \\
\hline
\end{tabular}

TABLE VII: Total energy for various methods for $\mathrm{SbH}$ at the equilibrium internuclear distance $e^{46}$ of $1.70187 \AA$.

\begin{tabular}{|c|c|c|c|}
\hline method & $r_{e}[\AA]$ & $\omega_{e}\left[\mathrm{~cm}^{-1}\right]$ & $\omega_{e} x_{e}\left[\mathrm{~cm}^{-1}\right]$ \\
\hline experiment & 1.702 & & \\
\hline experiment 44 & 1.711 & 1897 & \\
\hline experiment ${ }^{47}$ & & 1923 & 34.2 \\
\hline $4 c-\mathrm{SCF}_{\mathrm{f}}^{\mathrm{f}}$ & 1.705 & 2024 & 28.0 \\
\hline $4 \mathrm{c}-\mathrm{SCH}$ & 1.704 & 2043 & 38.1 \\
\hline 4c-MP2(16,81) $]^{f}$ & 1.693 & 2009 & 30.2 \\
\hline 4c-DMRG(16,14 $]^{\mathrm{f}} \mathrm{M}=2200$ & 1.737 & 1839 & 35.3 \\
\hline $4 c-\operatorname{CCSD}(16,81]^{\mathrm{f}}$ & 1.706 & 1945 & 32.6 \\
\hline $4 c-\operatorname{TCCSD}(16,14)^{\mathrm{f}} \mathrm{M}=2200$ & 1.706 & 1937 & 36.4 \\
\hline $4 \mathrm{c}-\operatorname{CCSD}(\mathrm{T})(16,81)^{\mathrm{f}}$ & 1.710 & 1916 & 35.3 \\
\hline
\end{tabular}

${ }^{\mathrm{f}}$ TWOFIT 4th order polynomial.

g VIBANAL 10th order polynomial.

TABLE VIII: Spectroscopic constants of ${ }^{121} \mathrm{SbH}$ obtained from 4c-TCCSD, compared with calculations and experimental work from the literature.

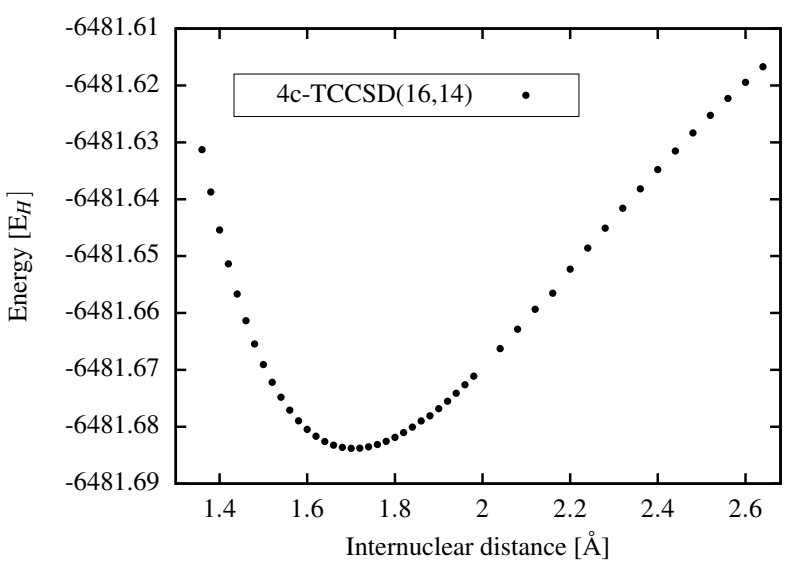

FIG. 6: Detail of potential curve of $\mathrm{SbH}$ near the equilibrium internuclear distance.

\section{v. CONCLUSION}

We have implemented the relativistic tailored coupled clusters method, which is capable of treating relativistic, strongly correlated systems both in terms of static and dynamical correlation. The aim was to show that compared with the previously published calculations, we can obtain results of equal quality with much smaller active space, i.e. at a fraction of computational cost. The results presented are promising. Even with a small active space, the new method showed comparable performance for TlH to DMRG with large CAS $(14,47)$. The optimal CAS size related to the energy minimum was determined with low cost DMRG calculations. 
Calculated spectroscopic properties of $\mathrm{TlH}$ agree with experimental values within the error bounds. The comparison with experimental spectroscopic constants for $\mathrm{AsH}$, which has stronger multireference character, has shown that TCCSD is able to describe such systems more accurately that CCSD, with a computational cost lower than $\operatorname{CCSD}(\mathrm{T})$.

\section{ACKNOWLEDGMENTS}

We thank developers of the Dirac program, in particular Dr. Lucas Visscher and Dr. Stefan Knecht, for providing access to the development version of the code and for helpful discussions. The work of the Czech team has been supported by the Czech Science Foundation Grant No. 18-24563S. Ö. Legeza has been supported by the Hungarian National Research, Development and Innovation Office (NKFIH) through Grant No. K120569, the Hungarian Quantum Technology National Excellence Program, project No. 2017-1.2.1-NKP-2017-00001. The development of the relativistic DMRG libraries was supported by the Center for Scalable and Predictive methods for Excitation and Correlated phenomena (SPEC), which is funded as part of the Computational Chemical Sciences Program by the U.S. Department of Energy (DOE), Office of Science, Office of Basic Energy Sciences, Division of Chemical Sciences, Geosciences, and Biosciences at Pacific Northwest National Laboratory. M. Mihály has been supported by the ÚNKP-19-3 New National Excellence Program of the Ministry for Innovation and Technology. Mutual visits with the Hungarian group have been partly supported by the Hungarian-Czech Joint Research Project MTA/19/04. Part of the CPU time for the numerical computations has been supported by The Ministry of Education, Youth and Sports from the Large Infrastructures for Research, Experimental Development and Innovations project "IT4Innovations National Supercomputing Center - LM2015070". Access to computing and storage facilities owned by parties and projects contributing to the National Grid Infrastructure MetaCentrum provided under the programme "Projects of Large Research, Development, and Innovations Infrastructures" (CESNET LM2015042), is appreciated.

${ }^{1}$ S. R. White, Physical Review Letters 69, 2863 (1992)

${ }^{2}$ S. R. White and R. L. Martin, The Journal of Chemical Physics 110, 4127 (1999)

${ }^{3}$ G. K.-L. Chan and M. Head-Gordon, The Journal of Chemical Physics 116, $4462(2002)$

${ }^{4}$ Ö. Legeza, J. Röder, and B. A. Hess, Physical Review B 67 (2003), 10.1103/physrevb.67.125114

${ }^{5}$ U. Schollwöck, Annals of Physics 326, 96 (2011)

${ }^{6}$ R. Olivares-Amaya, W. Hu, N. Nakatani, S. Sharma, J. Yang, and G. K.-L. Chan, The Journal of Chemical Physics 142, 034102 (2015)

${ }^{7}$ Y. Kurashige and T. Yanai, The Journal of Chemical Physics 135, 094104 (2011)

${ }^{8}$ M. Saitow, Y. Kurashige, and T. Yanai, The Journal of Chemical Physics 139, $044118(2013)$

${ }^{9}$ S. Wouters, N. Nakatani, D. V. Neck, and G. K.-L. Chan, Physical Review B 88 (2013), 10.1103/physrevb.88.075122

${ }^{10}$ T. Yanai and G. K.-L. Chan, The Journal of Chemical Physics 124, 194106 (2006)
${ }^{11}$ J. Ren, Y. Yi, and Z. Shuai, Journal of Chemical Theory and Computation 12, 4871 (2016)

${ }^{12}$ L. Veis, A. Antalík, J. Brabec, F. Neese, Ö. Legeza, and J. Pittner, The Journal of Physical Chemistry Letters 7, 4072 (2016)

${ }^{15}$ T. Kinoshita, O. Hino, and R. J. Bartlett, The Journal of Chemical Physics 123, 074106 (2005)

${ }^{14}$ O. Hino, T. Kinoshita, G. K.-L. Chan, and R. J. Bartlett, The Journal of Chemical Physics 124, 114311 (2006)

${ }^{15}$ S. Knecht, O. Legeza, and M. Reiher, The Journal of Chemical Physics 140, 041101 (2014)

${ }^{16}$ DIRAC, a relativistic ab initio electronic structure program (2018), T. Saue et al.http://www.diracprogram.org

${ }^{17}$ T. Saue and H. J. A. Jensen, The Journal of Chemical Physics 111, 6211 (1999)

${ }^{18}$ K. G. Dyall and K. Fægri Jr, Introduction to relativistic quantum chemistry (Oxford University Press, 2007).

${ }^{19}$ J. Thyssen, Development and Applications of Methods for Correlated Relativistic Calculations of Molecular Properties, Ph.D. thesis, Univeristy of Southern Denmark (2001).

${ }^{20} \mathrm{X}$. Li and J. Paldus, J. Comp. Phys. 107, 6257 (1997).

${ }^{21}$ D. I. Lyakh, V. F. Lotrich, and R. J. Bartlett, Chem. Phys. Lett. 501, 166 (2011).

${ }^{22}$ A. Melnichuk and R. J. Bartlett, J. Comp. Phys. 137, 214103 (2012).

${ }^{23}$ A. Melnichuk and R. J. Bartlett, J. Comp. Phys. 140, 064113 (2014).

${ }^{24}$ P. Piecuch, N. Oliphant, and L. Adamowicz, The Journal of Chemical Physics 99, 1875 (1993)

${ }^{25}$ P. Piecuch and L. Adamowicz, The Journal of Chemical Physics 100, 5792 (1994) https://doi.org/10.1063/1.467143

${ }^{20}$ K. Kowalski, The Journal of Chemical Physics 148, 094104 (2018)

${ }^{27}$ F. M. Faulstich, A. Laestadius, Ors Legeza, R. Schneider, and S. Kvaal, SIAM Journal on Numerical Analysis 57, 2579 (2019)

${ }^{28}$ L. Veis, A. Antalík, J. Brabec, F. Neese, Ö. Legeza, and J. Pittner, The Journal of Physical Chemistry Letters 8, 291 (2017).

${ }^{29}$ Ö. Legeza and J. Sólyom, Physical Review B 68 (2003), 10.1103/physrevb.68.195116

${ }^{30}$ U. Schollwöck, Rev. Mod. Phys. 77, 259 (2005).

${ }^{31}$ Ö. Legeza, R. Noack, J. Sólyom, and L. Tincani, in Computational ManyParticle Physics, Lecture Notes in Physics, Vol. 739, edited by H. Fehske, R. Schneider, and A. Weisse (Springer Berlin Heidelberg, 2008) pp. 653664.

${ }^{32}$ K. H. Marti and M. Reiher, Z. Phys. Chem. 224, 583 (2010).

${ }^{33}$ G. K.-L. Chan and S. Sharma, Ann. Rev. Phys. Chem. 62, 465 (2011).

${ }^{34}$ S. Wouters and D. Van Neck, Eur. Phys. J. D 68, 272 (2014).

${ }^{35}$ S. Szalay, M. Pfeffer, V. Murg, G. Barcza, F. Verstraete, R. Schneider, and Ö. Legeza, International Journal of Quantum Chemistry 115, 1342 (2015)

${ }^{36}$ T. Yanai, Y. Kurashige, W. Mizukami, J. Chalupský, T. N. Lan, and M. Saitow, Int. J. Quant. Chem. 115, 283 (2015).

${ }^{37}$ G. Moritz and M. Reiher, J. Chem. Phys. 126, 244109 (2007).

${ }^{38}$ K. Boguslawski, K. H. Marti, and M. Reiher, J. Chem. Phys. 134, 224101 (2011).

${ }^{39}$ D. Zgid and M. Nooijen, The Journal of Chemical Physics 128, 144115 (2008)

${ }^{40}$ S. Battaglia, S. Keller, and S. Knecht, Journal of Chemical Theory and Computation 14, 2353 (2018)

${ }^{41}$ Ö. Legeza, L. Veis, and T. Mosoni, "QC-DMRG-Budapest, a program for quantum chemical DMRG calculations,".

${ }^{42}$ F. M. Faulstich, M. Máté, A. Laestadius, M. A. Csirik, L. Veis, A. Antalik, J. Brabec, R. Schneider, J. Pittner, S. Kvaal, and Ö. Legeza, Journal of Chemical Theory and Computation 15, 2206 (2019)

${ }^{45}$ A. V. Titov, N. S. Mosyagin, A. B. Alekseyev, and R. J. Buenker, International Journal of Quantum Chemistry 81, 409 (2001)

${ }^{44}$ K. D. Hensel, R. A. Hughes, and J. M. Brown, J. Chem. Soc., Faraday Trans. 91, 2999 (1995)

${ }^{45} \mathrm{~K}$. Balasubramanian, Chemical Reviews 89, 1801 (1989)

${ }^{46}$ M. Beutel, K. Setzer, O. Shestakov, and E. Fink, Journal of Molecular Spectroscopy 179, 79 (1996)

${ }^{4 /}$ R.-D. Urban, K. Essig, and H. Jones, The Journal of Chemical Physics 99, 1591 (1993) 